Nouvelles perspectives en sciences sociales

Revue internationale de systémique complexe et d'études relationnelles

\title{
La faille et la brèche : réflexions sur un dépassement possible des controverses contemporaines en sociologie
}

\section{Claude Vautier}

Volume 9, numéro 1, novembre 2013

URI : https://id.erudit.org/iderudit/1024046ar

DOI : https://doi.org/10.7202/1024046ar

Aller au sommaire du numéro

\section{Éditeur(s)}

Prise de parole

ISSN

1712-8307 (imprimé)

1918-7475 (numérique)

\section{Découvrir la revue}

Citer cet article

Vautier, C. (2013). La faille et la brèche : réflexions sur un dépassement possible des controverses contemporaines en sociologie. Nouvelles perspectives en sciences sociales, 9(1), 289-317. https://doi.org/10.7202/1024046ar

\section{Résumé de l'article}

La sociologie n'en finit pas de confronter des positions selon lesquelles ce serait l'individu qui serait la source du tout social ou, au contraire, ce serait le système qui construirait les individus. Ces confrontations se poursuivent dans un débat autour de la synchronie ou de la diachronie des études. La sociologie des réseaux a marqué une avancée vers une réconciliation entre ces positions. Mais, pourrait-on dire, c'est une réconciliation a minima. Tout comme la théorie des systèmes complexes selon la mouvance du RNSC (Réseau national des systèmes complexes), si elle fait coexister une analyse structurale et une analyse individualiste, les catégories analytiques d'individu et de système restent nettement séparées et l'historicité du social n'est pas vraiment prise en compte.

Ce texte explore les possibilités de modélisation de l'inséparabilité des catégories individu, système et événement. Il tente de montrer que la tradition sociologique, jusqu'à nos jours, a manipulé ces catégories, les a appariées de diverses manières et que chaque courant théorique est analysable à travers une grille représentant le mode d'appariement de ces trois catégories. Il essaie de mettre en lumière le fait que ces combinaisons sont à la source de polémiques récurrentes et qui finissent par devenir largement stériles. Il le fait en développant l'idée qu'une approche relationnelle en sciences sociales peut être une voie de sortie de ces disputes en liant de façon intime les trois catégories précédentes. En leur accordant, dans la modélisation un niveau causal et résultant, soit de façon alternative, soit de façon simultanée, il permet leur circulation, comme dans un ruban de Möbius. Cette modélisation qui prend la forme d'un modèle trialectique noue, dans un périmètre élargi, ces grandes catégories de la sociologie sans donner prééminence à l'une sur les autres.
Ce document est protégé par la loi sur le droit d'auteur. L'utilisation des services d'Érudit (y compris la reproduction) est assujettie à sa politique d'utilisation que vous pouvez consulter en ligne.

https://apropos.erudit.org/fr/usagers/politique-dutilisation/ 


\section{La faille et la brèche : réflexions sur un dépassement possible des controverses contemporaines en sociologie ${ }^{1}$}

Claude Vautier

LEREPS

(n peut poser que la tradition sociologique, depuis Durkheim, Weber ou Simmel jusqu'à nos jours, mobilise fondamentalement trois catégories pour analyser le social. Il s'agit des catégories de système (recouvrant le social global), d'individu (l'agent, l'acteur, la personne...) et d'événement (introduisant l'histoire, l'historicité).

L'une des sources de la querelle des méthodes qui agita les sciences que l'on n'appelait pas encore sciences humaines et sociales $^{2}$ réside dans le fait que chaque courant de pensée, chaque auteur, focalise son étude sur l'une ou l'autre des trois catégories,

Il y a une querelle ancienne sur la place que peuvent prendre le « je " et le " nous " dans un article à vocation scientifique. Nous sommes tous sensibles à la dette que nous avons à l'égard des auteurs multiples qui nous ont éclairés, Je suis également conscient de tout ce que je dois à ceux que j'évoque dans ce papier et à beaucoup d'autres. Si j'opte pour la première personne du singulier, ce n'est pas par immodestie mais simplement pour assumer la responsabilité de mes propos.

2 Ce que l'on a appelé "Querelle des méthodes " débuta en 1883, date de publication de l'ouvrage de Karl Menger Recherches sur la méthode dans les sciences sociales et en économie politique en particulier, qui entraîna une réaction très négative de Gustav von Schmoller, chef de file de l'école historique allemande. 
considérant qu'il y en a une qui prédomine, qui est causale, tandis que les deux autres sont résultantes.

Dans les analyses classiques comme dans les courants s'étant développés dans les Sciences humaines et sociales par la suite, l'Individu (I), le Système (S) et l'Événement (E) sont possiblement de niveau causal (c) ou résultant (r). Peuvent se former ainsi des combinaisons où $\mathrm{I}, \mathrm{S}$ et $\mathrm{E}$ sont soit de niveau causal, soit de niveau résultant, soit en même temps de niveau causal et résultant. Il y a donc des basculements qui font qu'aucun des pôles n'est forcément ou causal ou résultant, mais que chacun peut être et causal et résultant.

Un tableau des huit occurrences possibles $\left(2^{3}\right)$, si on affecte des niveaux exclusifs (soit c, soit r) à chaque catégorie analytique, permet de balayer les diverses combinaisons entre les trois catégories selon qu'elles apparaissent de niveau causal ou résultant :

Tableau 1: Les huit combinaisons possibles entre les trois catégories analytiques selon leur niveau

\begin{tabular}{|c|c|}
\hline Situation & Symbolique \\
\hline I causal / S causal / E résultant & $\mathrm{Ic} / \mathrm{Sc} / \mathrm{Er}$ \\
\hline I causal / S résultant / E causal & $\mathrm{Ic} / \mathrm{Sr} / \mathrm{Ec}$ \\
\hline I causal / S résultant / E résultant & $\mathrm{Ic} / \mathrm{Sr} / \mathrm{Er}$ \\
\hline I résultant / $\mathrm{S}$ causal / $\mathrm{E}$ causal & $\mathrm{Ir} / \mathrm{Sc} / \mathrm{Ec}$ \\
\hline I résultant / S causal / E résultant & $\mathrm{Ir} / \mathrm{Sc} / \mathrm{Er}$ \\
\hline I résultant / S résultant / $\mathrm{E}$ causal & $\mathrm{Ir} / \mathrm{Sr} / \mathrm{Ec}$ \\
\hline I résultant / S résultant / E résultant & $\mathrm{Ir} / \mathrm{Sr} / \mathrm{Er}$ \\
\hline I causal / S causal / E causal & $\mathrm{Ic} / \mathrm{Sc} / \mathrm{Ec}$ \\
\hline
\end{tabular}

La réflexion qui suit tente de trouver dans les failles des approches traditionnelles et actuellement dominantes, une brèche vers un autre modèle théorique susceptible de permettre de dépasser les controverses incessantes autour de la prééminence de l'acteur ou du système, de la synchronie ou de la diachronie. 
Un regard sur les positions de divers auteurs dans le cadre de la question traitée ici fait apparaître le fait qu'aucun d'entre eux ne prend en compte la relation entre les trois catégories ${ }^{3}$.

Je tenterai de montrer dans la suite du texte que cette insuffisance se double d'une autre tout aussi préjudiciable aux analyses, la non prise en compte des relations entre chaque terme et la relation existant entre les deux autres. D'une certaine manière (et sans épiloguer sur le terme "paradigme "), c'est d'une autre approche paradigmatique qu'il s'agit.

Cet article est constitué autour de trois intentions principales.

D’une part évoquer rapidement la lente évolution des SHS vers une approche du social par les relations, dont les applications SMA (Sytèmes Multi Agents), la théorie des réseaux ou, plus nettement encore, la sociologie dite relationnelle d'Emirbayer ou Donati, sont de bons exemples (La valse des paradigmes).

D'autre part, exposer un nouveau paradigme "relationnel» pour les sciences humaines et sociales. Je voudrais essayer de fonder sur un plan théorique, à partir des positions de certains auteurs, anciens ou contemporains, issus des SHS ou d'autres disciplines, l'idée que la séparation à l'œuvre dans la représentation d'un système social comme système d'acteurs peut être contre-productive dans la recherche de compréhension de ce qu'est une société et de la façon dont elle se transforme. Je mobiliserai, pour ce faire, les concepts de discontinuité, de continuité et d'hologrammie pour définir la notion de « champ relationnel » (Continuité et discontinuité; Champ de relation ou champ relationnel?; Champ relationnel et hologrammie).

Enfin, proposer la généralisation d'un modèle relationnel contemporain pour développer une théorie générale des SHS dans laquelle, non seulement, les approches individualistes et holistes sont nouées plutôt qu'opposées, mais peut-être surtout, dans laquelle la relation elle-même ne sépare plus les termes qu'elle est censée relier, mais est consubstantielle à ceux-ci. Une telle " théorie générale " ne prétend pas à être exclusive, ni

3 Une étude empirique en cours qui sera présentée dans un article ultérieur confirme cette appréciation qui peut, d'ailleurs, sembler assez banale. 
exhaustive. Elle tend à éviter les oppositions frontales entre des approches légitimes mais parcellaires en les réunissant (Le modèle trialectique de Simon Laflamme; Le modèle trialectique hologrammique).

\section{La valse des paradigmes}

D'où viennent ces basculements de paradigmes, ces substitutions de groupements de paradigmes que sont les «épistémès » de Foucault? On ne sait pas très bien, mais on sent intuitivement qu'on a affaire à des mouvements primordiaux qui bousculent toute la sensibilité humaine, qu'il s'agisse de l'intellection, de l'art, de l'affectivité et, finalement, de la façon dont les hommes vivent et se représentent leur vie en société écrivait Yves Barel en $1977^{4}$.

Il y a aujourd'hui d'autres basculements de paradigmes, d'autres substitutions de groupements de paradigmes dont on sent bien, intuitivement, qu'ils correspondent à des mouvements primordiaux, peut-être à des tentatives, parfois quelque peu désespérées, de donner un ou des sens à ce qui semble en avoir trop ou, ce qui revient au même, n'en avoir pas.

Voici, par exemple (mais c'est un exemple qui me semble lui-même paradigmatique), un débat nourri autour de la question de la place de la relation en sociologie. Je ne veux pas évoquer les questions relatives à l'interactionnisme, symbolique ou non, ni celles qui ont trait à l'émergence de phénomènes sociaux à partir de ces interactions... Ce sont, me semble-t-il, de vieilles lunes qui nous ont aidés à comprendre certains des phénomènes du social mais nous entravent plutôt aujourd'hui si on ne les met en perspective dans un autre cadre de pensée et dans une autre terminologie $e^{5}$.

Le débat dont je parle est celui qui oppose ceux pour qui la relation est forcément " relation entre des choses, des objets, des phénomènes ", est donc un medium comme dit Donati, et ceux

$4 \quad$ Yves Barel, «L'Idée de système dans les sciences sociales ", Esprit, n 1, janvier 1977, p. 70.

5 Déjà, Joseph Schumpeter pouvait écrire, dans son Histoire de l'analyse économique, Paris, Gallimard, 1954, parution posthume, que cette querelle des méthodes était " un dialogue de sourds totalement inutile». 
qui professent que la relation est première, et donc, non pas medium, mais "point de vue à partir duquel la sociologie peut saisir ses objets dans leur nature sociale ", comme l'écrit aussi Donati ${ }^{6}$.

Il me semble qu'aucun sociologue contemporain n'est en mesure de nier l'intérêt d'étudier les relations dans un système sociétal. Qu'aucun non plus ne peut mettre en doute qu'il y a eu progrès à considérer que toute "structure " sociale est une émergence des interactions entre les composants d'une telle «structure». De même, l'exploitation de ce principe selon lequel un tel système peut être décrit en termes de réseaux me semble permettre une exploration plus fine de tels systèmes, dans la mesure où l'opérationnalité de cette posture est assurée avec une bonne dose de succès dans la capacité de maîtrise que nous pouvons obtenir sur ces systèmes.

Maintenant, une chose est de décider que les relations entre les pôles d'un réseau représentatif d'un système permettent de comprendre, modéliser, manipuler le système en question; autre chose est de dire que ce n'est pas la relation entre des pôles qui nous intéresse, mais le maillage relationnel, la relation en tant que champ.

Ce que je souhaite interroger ici, c'est ce basculement qui tente de se faire jour en remplaçant l'idée qu'un système social peut se décrire à partir de relations entre des "choses " (collectivités humaines, individus, objets, règles...) par celle qu'un tel système peut se dessiner comme étant avant tout un champ de relation ayant ses caractéristiques propres pouvant s'étudier indépendamment des caractéristiques des " choses " que l'on pense pouvoir dire " en relation ", notamment la psyché des acteurs humains, leur type de comportement, l'intentionnalité de ceux-ci, leur type de rationalité, etc. ${ }^{7}$

6 Pierpaolo Donati, "La Relation comme objet spécifique de la sociologie ", Revue du MAUSS, vol. 2, n 24, 2004, p. 233-254, en ligne sur <www.cairn. info/load_pdf.php?ID_ARTICLE=RDM_024_0233>, consulté le 20 juillet 2012.

7 Dans l'introduction au $n^{\circ} 140$ / 2008 de la Revue européenne des sciences sociales, François Chazel et Jacques Coenen-Huther écrivent : "Jacques 
Sous cet angle, la question correspond (dans son esprit mais aussi dans ce qu'elle a à voir avec la notion de système) à celle que posait Yves Barel dans l'article précité en définissant le concept de "système " comme " une machine à problème ".

Le débat qui fait irruption autour du statut de la relation dans les sciences sociales peut être interprété également comme " machine à problème » ou comme problématique « au sens fort, c'est-à-dire une certaine façon de découvrir des problèmes qui pourraient ne pas être aperçus autrement ${ }^{8}$ ».

Dans la batterie d'outils permettant aux sciences sociales d'étudier empiriquement diverses caractéristiques des sociétés (les processus de décision, les phénomènes de conflit et de délibération, par exemple), l'idée que les individus sociaux et les collectifs qu'ils forment représentent des entités sociales dont les « comportements " donnent à voir quelque chose de la statique et de la dynamique (régulation homéostatique et dynamique) des sociétés est une ressource efficace. La mise en relation, dans des modèles multi agents par exemple, d'entités virtuelles simulant des comportements sociaux permet une exploration que l'on peut juger prospective de situations sociétales diverses?. Elle permet aussi, par sa proximité factuelle avec notre vision intuitive de ce qui se passe dans les sociétés humaines, de mettre en œuvre des jeux de rôles sociaux et des modes de modélisation participative

Coenen-Huther propose d'axer la théorie générale sur un programme de sociologie formelle dans la tradition de Simmel, Dupéel et Bouglé [...] Il s'agit d'une sociologie relationnelle dont l'unité n'est pas l'individu mais bien la situation d'interaction ou, si l'on préfère, le rapport social. Cette conception relationnelle du social permet de surmonter l'opposition qu'on peut tenir pour surannée entre le holisme et l'individualisme ", François Chazel et Jacques Coenen-Huther, "La Théorie sociologique générale en question ", Revue européenne de sciences sociales, tome XLVI, 2008, n 140, p. 5-14.

8 Yves Barel, op. cit., p. 76.

9 C'est le cas du modèle "Soclab » conçu par une équipe d'informaticiens et de sociologues de l'Université de Toulouse 1 Capitole et utilisé dans le cadre d'un contrat européen intitulé Concert'Eau. Voir sur le premier point Pascal Roggero et Christophe Sibertin-Blanc, " Quand des sociologues rencontrent des informaticiens : essai de formalisation, méta modèle, modélisation et simulation des systèmes d'action concrets ", Nouvelles perspectives en sciences sociales, vol. $3, \mathrm{n}^{\circ} 2,2008$, p. 41-81; sur le second point, on consultera avec profit le site concerteau.ecobag.org/ 
où les individus et/ou les collectifs en interaction sont à la fois les objets et les sujets de la modélisation dans une boucle récursive qui dépasse les schémas de classification et de causalité linéaires ${ }^{10}$. L'association de ces recherches à la théorie des réseaux, la théorie des réseaux elle-même, sont autant de voies fécondes pour tenter de " mettre un peu d'ordre " dans nos perceptions du monde social et essayer d'y obtenir une maitrise permettant d'en améliorer l'efficacité pour les humains (dans une perspective humaniste) et pour les institutions sociétales (entreprises, administrations...) dans une perspective plus terre à terre, voire plus cynique.

Pourtant, de la même manière que Barel voyait dans le PPBS et la $\mathrm{RCB}^{11}$ " le symétrique exact de [cette] recherche causale classique $^{12}$ " en même temps qu'il voyait dans l'idée de système (et par extension de rationalisation) un risque de systématisa$\operatorname{tion}^{13}$, on peut voir l'appareillage contemporain évoqué ci-dessus comme la pointe avancée d'une technologie de recherche sociologique incontournable aussi bien que, contradictoirement, comme un nouvel avatar de la position phénoménologique ${ }^{14}$ tentant de garder une place de paradigme dominant dans les sciences humaines et sociales.

10 Voir par exemple, William's Daré, Christine Fourrage et Ibrahima Diop Gaye, "Positionnement des sociologues dans la démarche de modélisation Domino ", Nouvelles perspectives en sciences sociales, vol. 2, n 2, 2007, p. 103126.

11 Planning Programming Budgeting System et Rationalisation des Choix Budgétaires, tentatives de rationalisation des décisions, notamment publiques, en vogue au cours des années 1960-1970 en France.

12 Yves Barel, op. cit., p. 75.

13 Yves Barel, op. cit., p. 69.

14 Le terme "phénoménologie " est pris ici dans son sens husserlien, dans la mesure où il a un rapport direct avec l'intentionnalité de la conscience. En termes de méthode sociologique, cela conduit à s'interroger sur la catégorie " conscience » et sur la catégorie "intentionnalité ». La présente recherche essaie de s'émanciper de ce détour par la conscience des acteurs et pose que la question de l'intentionnalité ramène à celle de la rationalité et laisse ainsi de côté tous les actes non intentionnels et non rationnels. 


\section{Continuité et discontinuité}

Cet aspect du débat contemporain autour de la relation me semble renvoyer à un débat plus ancien ayant eu lieu dans une autre discipline que les sciences humaines et sociales au début du $\mathrm{XX}^{\mathrm{e}}$ siècle. Cette révolution apparaît notamment dans les contradictions et les proximités entre la théorie de la relativité et la théorie quantique. Le physicien anglais David Bohm l'exprime ainsi :

La relativité exige une stricte continuité, un déterminisme strict et une stricte localité; pour la mécanique quantique, c'est exactement le contraire : discontinuité, non-déterminisme et non-localité [...] Ce qu'elles ont de commun, c'est cette idée de complétude ininterrompue de l'univers ${ }^{15}$.

Cette façon de réconcilier les deux grandes théories sur lesquelles s'est construite la physique contemporaine présente un intérêt qui déborde les sciences de la nature. Bohm développe tout au long du colloque, dont sont tirés ces extraits, l'idée que le monde est holographique ${ }^{16}$, c'est-à-dire, littéralement, que le monde " écrit " le " tout " ${ }^{17}$. Cette qualité holographique permet, selon lui d'expliquer que le monde soit à la fois discontinu et continu. Je passe sur l'argumentation qui recourt à la notion de structure "impliée » et "dépliée » et qu'il traduit ainsi :

Prenons un électron qui se déploie [...] à une position particulière, puis qui s'y replie à nouveau, tandis qu'un autre se déploie à côté de lui, puis se replie encore, et puis un autre et un autre... jusqu'à ce que, graduellement, il nous semble voir la trace d'un seul électron. On observe ici la discontinuité, car les points du déploiement n'ont pas besoin d'être

15 David Bohm, La Danse de l'esprit ou le sens déployé, La Varenne-Saint Hilaire, Seveyrat, non daté, p. 15-16. Ce texte correspond aux actes d'un colloque tenu dans les Coswold Hills, apparemment en février 1985. Le soulignement est de moi.

16 Je rappelle que l'hologramme est défini ainsi par Morin : «Les parties peuvent être singulières ou originales tout en disposant des caractères généraux et génériques de l'organisation du tout; les parties peuvent être douées d'autonomie relative; elles peuvent établir des communications entre elles et effectuer des échanges organisateurs; elles peuvent être éventuellement capables de régénérer le tout ", Edgar Morin, La Méthode 3. La connaissance de la connaissance, Paris, Seuil, 1986, p. 102. 
continus. Et l'on comprend pourquoi il peut y avoir à la fois discontinuité et continuité - les qualités des ondes - dans le déploiement ${ }^{18}$.

À mon avis, profondément, la métaphore exprime une idée fondamentale. Les éléments discontinus sont en fait insérés dans un tout qui leur donne son sens (non pas celui des éléments, mais celui du système). À y regarder d'un peu plus près, une telle image est heuristique pour ce que signifie l'existence simultanée pour un même objet de continuité et de discontinuité. Dans le langage de Bohm, que reprennent divers sociologues, ceci constitue un "champ " continu sans lequel ni la discontinuité (" réelle »?), ni la continuité (" apparente »?) n'existeraient. Autrement dit, sans ce tout qui est un agencement de relations entre les éléments discrets et lui-même, ces éléments n'auraient tout simplement pas de sens. Ce qui implique qu'il n'y a peut-être pas de séparation, ni ontologique ou principielle, ni conceptuelle entre continuité et discontinuité.

\section{Champ de relations ou champ relationnel?}

Ces quelques remarques ne sont pas à même d'éliminer la perplexité du chercheur confronté à ce type de problème qui consiste à décider si un système est un ensemble d'éléments discrets en relation ou s'il est un ensemble de relations en relation ou encore un champ de relations, voire un champ de relation (sans " $\mathrm{s} »)^{19}$, ce que je nomme, après d'autres, mais dans un sens particulier qui sera précisé plus bas, "champ relationnel ».

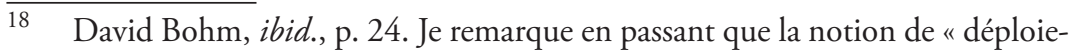
ment " qu'utilise David Bohm reste mal déterminée comme la proposition " un électron qui se déploie ». Mais, pour l'instant, dans le cadre de cette réflexion, je ne souhaite pas discuter ces questions.

19 Dans un premier temps, je peux distinguer l'expression avec « $\mathrm{s}$ » de celle qui n'en comprend pas en disant qu'un " champ de relations (avec s) » correspond plutôt à la conception classique de relation " entre des choses » et l'expression " champ de relation (sans « $s$ ") " veut représenter un monde où ce n'est pas ce qui est en relation (les « choses ») qui compte, mais l'opération "relation » qui permet de constituer un monde ou, peut-être plus précisément, où « le champ de relation " est la métaphore du système sociétal et la matrice de sa modélisation. 
Je voudrais remarquer que, de la même manière que la théorie de la relativité et la théorie quantique, avec les contradictions qui les opposent, ont cheminé en apportant leurs lots de connaissances respectives et partiellement mutualisées, les diverses positions dans le champ sociologique ont toute légitimité à s'exprimer. Chaque chercheur a également légitimité à se positionner comme il l'entend et en fonction de ses propres objectifs (que d'autres les jugent pertinents ou non, c'est alors affaire de débat argumenté dans la communauté).

Pour ce qui me concerne, j'ai mis un pied dans au moins deux postures apparemment contradictoires. J'ai participé à des travaux fondés sur des Systèmes Multi Agents, acceptant de considérer que l'on peut décrypter un système sociétal en mettant en scène des acteurs sociaux en relation sous les conditions de leurs contraintes, de leurs buts et rationalités (donc de leur psyché), bien qu'ici il se soit agi d'acteurs virtuels, d'entités informatiques; d'autre part, j'ai tenté de traiter un système sociétal à travers des concepts systémiques en relation les uns avec les autres, travail pouvant être jugé comme étant à mi-chemin entre individualisme et holisme méthodologique. Ses fondements avaient leurs racines dans les acteurs dont les représentations et les discours étaient interrogés; mais, dans la modélisation, ce n’étaient plus des acteurs sociaux qui étaient en relation, mais des caractéristiques analytiques du système, informées par les réponses des acteurs. Mon sentiment était alors que si les acteurs étaient sources de productions sociales, les hypothèses nécessaires sur leurs comportements posaient un problème insoluble : la rationalité qu'il faut bien leur prêter n'épuise jamais, quel que soit le raffinement qu'on y mette, la complexité du « réel » observable et c'est moins l'ajout de nouvelles caractéristiques successives ${ }^{20}$ qui peut sauver

20 À la manière décrite par Imre Lakatos, lorsque la théorie est en défaut, on ajoute des hypothèses supplémentaires dans l'un des cercles protecteurs de celle-ci pour en sauver le cœur : la rationalité est d'abord instrumentale, puis elle est procédurale, puis elle est axiologique, avant de devenir émotive (voir le concept d'émorationalité proposé par Simon Laflamme, dans Communication et émotion. Essai de microsociologie relationnelle, Paris, Paris, L'Harmattan, coll. "Logiques sociales ", Paris 1995... Quel que soit l'intérêt (et la légitimité) de ces hypothèses pour se rapprocher de l'observation phénoménale, l'exercice 
le modèle qu'un nouveau regard sur la question posée. Muni de ce sentiment, je me suis donc interrogé sur l'intérêt de la sociologie relationnelle, telle que proposée initialement par Bajoit, Donati ou Emirbayer ${ }^{21}$ et, plus récemment et, me semble-t-il, plus radicalement, par Simon Laflamme ${ }^{22}$, qui me paraissait renouveler le questionnement. Tout en admettant l'intérêt des diverses démarches possibles, c'est sur cette dernière posture (relationnelle) que je souhaite mettre l'accent.

Mon objectif est de tenter de convaincre que l'on peut échapper aux dilemmes qui produisent des débats dans les sciences sociales : individualisme vs holisme, rationalité vs non rationalité, intentionnalité $v s$ non intentionnalité, ainsi que, dans la sociologie qui se dit relationnelle, « relations entre » vs « champ relationnel».

peut s'avérer sans fin : Pareto délaissait les « actions non logiques » que Morin appelle à réintégrer en disant que l'homme est " homo sapiens-demens "; l'idéologie est ramenée à la rationalité par certains (Boudon, par exemple, et ses « bonnes raisons "), tandis que d'autres veulent intégrer les transformations que la pensée idéologique entraîne sur la rationalité; chez les économistes, les anticipations furent rationnelles mais ne le sont plus...

21 Notamment dans : Guy Bajoit, Pour une sociologie relationnelle, Paris, Presses universitaires de France, 1992; Pierpaolo Donati, «La Relation comme objet spécifique de la sociologie ", op. cit.; Mustafa Emirbayer, "Manifesto for a Relational Sociology ", American Journal of Sociology, vol. 103, n² 2, 1997 , p. 281-317. Concernant Bajoit, il me semble que sa sociologie est moins " relationnelle " que centrée sur les " relations sociales entre " : " la relation sociale, écrit-il, peut être définie comme un échange entre deux acteurs qui éveille en eux des attentes... ", Guy Bajoit, " Le Concept de relation sociale ", Nouvelles Perspectives en sciences sociales, vol. $5, \mathrm{n}^{\circ} 1,2009$, p. 51.

22 Claude Vautier, "La Longue marche de la sociologie relationnelle », Nouvelles perspectives en sciences sociales, vol. 4, $\mathrm{n}^{\circ} 1$, 2008, p. 77-106. Parmi les textes de Simon Laflamme, celui où la sociologie relationnelle trouve son premier exposé abouti est : La Société intégrée. De la circulation des biens, des idées et des personnes, New York, Peter Lang, 1992. Voir aussi, Mélanie Girard, "Éléments de critique des théories de l'action ", Nouvelles perspectives en sciences sociales, vol. 3 , $\mathrm{n}^{\circ}$ 1, 2007, p. 47-60 et Contribution à la critique des théories de l'action. Intention et émoraison, Thèse de doctorat en sociologie, Toulouse, Université des Sciences Sociales, Toulouse 1 Capitole, 2009; Pierre Bouchard, "Théorie de l'action et parcours de vie ", Nouvelles perspectives en sciences sociales, vol. 1, $\mathrm{n}^{\circ}$ 2, 2006, p. 67-114; Roger Gervais, Presse et Mondialisation : étude comparée franco-canadienne, Thèse de doctorat, Université de Toulouse 1 Capitole, 2009. 
Dans ce cadre, il me semble que la première question qui vient à l'esprit est : " que signifie le terme relation si on n'exhibe pas quelques éléments qui sont en relation?".

Pour tenter de répondre à cela, sans doute faut-il revenir à l'idée fondamentale qu'un système est avant tout une construction discursive et/ou empirique. Ce que nous appelons «système " n'existe pas dans la nature mais seulement dans notre esprit comme moyen de préhension du réel perçu et inaccessible directement, sans médiation de notre cerveau (ou de " nos cerveaux interconnectés ", comme dit Edgar Morin) et d'un langage, d'une sémantique. La notion de "champ relationnel $»^{23}$ est une image commode et heuristique pour dire, d'une part, l'impossibilité de concevoir un monde social en dehors de la notion générique de "relation ", d'autre part, l'intérêt de raisonner en utilisant le mot « relation » sans le faire suivre du terme " entre ». Il s'agit d'une métaphore et, au sens profond du terme, d'une " modélisation " du social. Cette modélisation doit s'appuyer sur des principes qui rendront praticable la métaphore et qui lui permettront, sur un plan plus théorique, de toucher au sens.

Parmi ces principes, je veux relever celui d'hologrammie et montrer que la sociologie que l'on s'accorde à peu près à nommer " relationnelle » a avantage à le mobiliser.

\section{Champ relationnel et hologrammie}

Les dictionnaires courants donnent du terme « holographie » une définition du type : "Méthode d'enregistrement et de reproduction photographique fondée sur la superposition ${ }^{24}$ de deux faisceaux laser" (Nouveau Littré, édition 2004). Barel, lorsqu'il veut s'interroger sur la topologie des systèmes, parle de "systèmes ou parties de systèmes [qui] se recouvrent partiellement les uns les autres, se superposent ${ }^{25}$ ". J'effectue un rapprochement autour du mot "superposition " parce que ce dernier me semble être

23 Désormais, je ne traiterai, sauf indication explicite, que de la notion de champ relationnel. Si j'utilise le terme " champ de relation » (sans «s ») je ferai dans ce cas l'économie de la parenthèse.

24 C'est moi qui souligne.

25 Yves Barel, op. cit., p. 78-79. 
une clé de la compréhension de cette idée, a priori peu compréhensible, selon laquelle on pourrait parler de relation sans parler d'êtres en relation (ou, avec un affreux barbarisme, "relationnés ").

D'une certaine manière, ce que j'appelle « champ relationnel » tient à la qualité que je prête à ma perception du monde. C'est, pour le dire vite, le caractère hologrammatique (selon le terme d'Edgar Morin) du monde. Morin, tout en faisant référence aux travaux de David Bohm, écrit, dans le tome 3 de La Méthode:

Le principe hologrammatique généralisé que nous allons formuler ici dépasse le cadre de l'image physique construite par laser. C'est peut être un concept cosmologique clé. De toute façon, il concerne la complexité de l'organisation vivante, la complexité de l'organisation cérébrale et la complexité socio-anthropologique. On peut le présenter ainsi : le tout est d'une certaine façon inclus (engrammé) dans la partie qui est incluse dans le tout ${ }^{26}$.

Cette idée d'un caractère hologrammatique du monde en général et du monde social en particulier m'est initialement apparue de nature à éclaircir le sens de la notion de "champ relationnel ». Sans doute en faisant le lien entre les notions de continuité et discontinuité, d'une part, et les notions de tout et de parties imbriquées dans des hiérarchies enchevêtrées ${ }^{27}$, d'autre part. Sans doute en essayant de montrer que les notions de " relation entre " (les parties) et de " champ relationnel » (le tout et les parties entremêlés) correspondent à cette coexistence de discret et de continu pour un même phénomène, dans un même lieu, en un même temps et a simultanément quelque chose à voir avec la question de l'hologrammie.

Cette idée peut-elle résoudre quelques-unes des questions cruciales qu'il faut bien aborder. Parmi elles, l'une me semble devoir être évoquée ici. Cornelius Castoriadis l'énonce ainsi :

\footnotetext{
$26 \quad$ Edgar Morin, op. cit., p. 101-102.

27 À ma connaissance, ce terme a été proposé initialement par Douglas Hofstadter, Gödel, Escher, Bach. Les brins d'une guirlande éternelle, New York, Basic Books Inc. Publishers, 1979 (traduction de Jacqueline Henry et Robert French, InterÉditions, 1985).
} 
Nous sommes placés devant cette exigence, apparemment insoutenable, de penser des " relations " entre des " termes " qui ne soient pas des entités discrètes, séparées, individualisables, plus encore, de considérer la dyade "termes-relations ", telle qu'elle se présente chaque fois à un niveau déterminé, comme impossible à saisir à ce niveau-là indépendamment des autres ${ }^{28}$.

L'une des voies de résolution possible de l'énigme ainsi posée par Castoriadis se trouve peut-être dans la fin de la phrase (celle que j'ai soulignée par des italiques) : ne serait-ce pas une question de " niveau "? J'évoquais ci-dessus la notion de " hiérarchie enchevêtrée " proposée par Hofstadter. Ce dernier évoque l'existence d'un " niveau intouchable » qui est celui des conventions qui régissent le modèle et ne peut, en théorie être modifié ${ }^{29}$. Évoquant une situation de jeu d'échec où chaque coup modifierait la règle du jeu, Hofstadter écrit : "Les coups changent les règles, les règles déterminent les coups, et tourne le manège ! Il y a toujours des niveaux différents, mais la distinction entre les "plus bas" et les "plus hauts" s'est estompée ${ }^{30}$.

Cependant, le terme "hiérarchie ", même suivi du mot " enchevêtrée " peut induire en erreur. Ce que je veux mettre en évidence, c'est justement qu'il n'y a pas de hiérarchie. J'interprète ainsi le terme de Hofstadter : l'enchevêtrement des niveaux annule la hiérarchie ${ }^{31}$. Là où l'on croit voir une différence de

28 Cornelius Castoriadis, Les Carrefours du labyrinthe, tome 1, Paris, Seuil, 1978, p. 260. C'est moi qui souligne.

29 "Il y a un niveau intouchable, que nous appellerons le niveau I, auquel se trouvent les conventions d'interprétation, et aussi un niveau enchevêtré, le niveau $E$, qui est celui de la Hiérarchie Enchevêtrée. Ces deux niveaux forment donc une petite hiérarchie : le niveau I détermine ce qui se passe au niveau E, mais le niveau E ne peut affecter le niveau I. Peu importe que le niveau E soit lui-même une Hiérarchie Enchevêtrée; il est toujours régi par un ensemble de conventions qui lui sont extérieures. Et c'est là le point important ", Douglas Hofstadter, Gödel, Escher Bach, op. cit., p. 775. Cette formulation est fondée, explicitement dans le texte, sur le théorème d'incomplétude de Gödel. D'une certaine manière, elle ignore la position de Lakatos sur le changement du corps d'hypothèses.

$30 \quad$ Ibid., p. 775

31 De ce fait, le terme " hiérarchie enchevêtrée " pourrait être considéré comme un oxymore. Mais, en fait, il s'agit plutôt d'une hiérarchie qui change constamment, chacun des termes hiérarchisés ayant le potentiel d'être en 
niveau, en fait il n'y en a pas, puisque le niveau empirique auquel interviennent les catégories analytiques du modèle est toujours possiblement indéfini. Il existe pour chacune d'entre elles un champ de possibilité dans lequel les niveaux ne sont que temporairement fixés, dans lequel ils sont successifs et/ou simultanés. Ce que j'essaie de dire, comme Hofstadter, me semble-t-il, c'est que la hiérarchie apparente est en fait une illusion qui, d'ailleurs, est source de paradoxe, au sens de Barel. En d'autres termes, c'est la construction du modèle qui conduit à fixer, même provisoirement, comme pas dans le raisonnement, des niveaux hiérarchiques : le concept de hiérarchie enchevêtrée n'est autre qu'une représentation possible du caractère hologrammatique de la vision du monde qui est dessinée dans le modèle. Et ce principe hologrammatique est un "principe d'intelligibilité32 " qui se situe au niveau "intouchable ", selon Hofstadter. Comme l'écrit Edgar Morin : "C'est surtout pour essayer de comprendre la représentation, l'inscription en mémoire et la remémoration que l'on peut, à la suite de Pribram, faire appel au principe hologrammatique $^{33}$ ».

Ces réflexions rejoignent, a contrario, l'expression, dans le langage vernaculaire et dans le sens commun, de l'impossibilité d'une société où les êtres sociaux ne seraient pas séparables, distinguables les uns des autres, ne seraient donc pas « en relation " les uns avec les autres, mais seraient en quelque sorte eux-mêmes et l'ensemble des autres?

Le langage des choses quotidiennes n'est à l'évidence pas adapté pour évoquer ce qui vient d'être écrit ci-dessus. Mais le langage scientifique qu'utilise Castoriadis lui-même n'est pas exempt d'une certaine inadéquation, par force pourrait-on dire :

position supérieure ou inférieure selon le moment (diachronie) ou la situation du système (synchronie)... L'idée selon laquelle le terme "enchevêtrée " annule la hiérarchie n'est donc qu'une approximation. La hiérarchie existe pour un instant donné ou bien plusieurs hiérarchies existent en même temps selon la façon dont on observe le système.

32 Edgar Morin, op. cit., p. 98.

33 Ibid., p. 103. 
Il n'est pas possible, visiblement, de penser la société comme " composition » à partir d'éléments qui logiquement ou réellement lui préexisteraient : on ne pourrait " composer " une société, si déjà l'expression a un sens, qu'à partir d'êtres déjà sociaux, c'est-à-dire qui portent déjà le social en eux-mêmes. Pas plus qu'il n'est possible d'appliquer ici le schème qui, tant bien que mal, semble applicable à d'autres domaines, à savoir qu'il émerge au niveau d'une " totalité » des propriétés nouvelles qui n'existent pas ou qui n'ont pas de sens au niveau des " composants". [...] Il n'y a aucun sens à considérer que langage production, règles sociales sont des "propriétés " qui émergent dès que l'on met ensemble un nombre suffisant d'individus; ces individus sont inexistants et inconcevables hors ou avant ces prétendues " propriétés collectives » - et n'y sont pas non plus réductibles ${ }^{34}$.

C'est par touches successives que ce dernier cerne son objet en nous disant tout autant l'impossibilité de penser la société comme agglomérat d'individus "inexistants et inconcevables hors ou avant ces prétendues "propriétés collectives" ", ou comme totalité siège d'émergences n'ayant " pas de sens au niveau des "composantes" ", en bref la contradiction apparemment indépassable d'une représentation de la société et comme un ensemble d'éléments discrets, et comme un tout unifié et comme un ensemble de relations entre des objets qui n'existent pas en dehors de la relation et comme ensemble de relations qui ne sont relations entre rien...

Castoriadis nous donne aussi quelques pistes que l'on peut, me semble-t-il, interpréter en termes d'hologrammie :

est évacuée la question : l'inhérence, l'empiètement, la réversibilité, le passage, le et de : signe et sens, ce qu'on ne saurait, même avec des précautions infinies, appeler leur relation qu'en se trompant déjà, puisque la relation a figé deux termes comme extérieurs l'un à l'autre ou en tout cas séparables, et qu'une fois cette séparation commise il sera à jamais impossible de ressaisir ce dont il s'agit, pour les mêmes raisons qui rendent impossible de reconstruire un monde avec pour seuls et dérisoires instruments un Ego ou une sémiosis pure ${ }^{35}$.

"L'inhérence ", "l'empiètement »... l'impossibilité de penser la relation si ce n'est entre, etc., tout cela réfère à la fois à la

34 Cornélius Castoriadis, Les Carrefours du Labyrinthe, op. cit, p. 258.

35 Cornélius Castoriadis, Ibid., p. 165. 
question de l'inséparabilité indicible entre les acteurs et le système, à la question du continu et du discontinu et, en même temps, à celle du caractère hologrammatique où la partie est consubstantielle au tout qui se trouve lui-même dans chaque partie. Cela ne nous dit-il pas qu'il est possible d'imaginer de la relation qui ne soit entre, puisque les parties seraient consubstantielles au tout? Et que " la relation », et plus précisément le " champ relationnel ", pourrait être, dans cette construction, en tant que catégorie analytique ou théorique mais non empirique, l'équivalent de ce "filet de relations humaines " dont parle Norbert Elias ${ }^{36}$ et qui constitue pour lui le substrat de toute construction socio-historique de l'individu. Et l'on remarquera, si l'on veut bien pour l'instant accepter cette proposition, qu'une telle approche relationnelle hologrammatique n'élimine pas les individualités, les éléments séparés ou séparables, mais les tient de manière égale pour ce substrat révélateur des configurations du champ de leur propre construction identitaire.

Le caractère hologrammatique d'un modèle sociologique relationnel est d'abord " hors champ ", c'est une perspective que l'on se donne pour prendre en compte les paradoxes qui apparaissent dans le modèle. Il est le niveau " auquel se trouvent les conventions d'interprétation ${ }^{37}$ ", le niveau du " grand tableau universel " : "Ce n'est pas parce qu'aucun de nous ne pourra jamais reculer suffisamment pour voir le " grand tableau universel " qu'il faut oublier qu'il existe ${ }^{38}$. J'interprète le concept de " grand tableau universel " comme le cadre postulatif grâce auquel nos constructions de modèles prennent du sens.

Cependant, le caractère hologrammatique du modèle intervient aussi au sein de la hiérarchie enchevêtrée, comme " mode

$36 \quad$ Norbert Elias, La Société des individus, Paris, Fayard, 1991 [1987], p. 72 : "Et c'est l'ordre de ce réseau permanent qui ne commence nulle part, c'est l'histoire de ses relations qui font la nature et la forme de la personne individuelle. Même la nature et la forme de sa solitude, même ce que l'individu ressent comme son "intériorité" reçoivent l'empreinte de cette histoire l'empreinte du filet de relations humaines dont il est l'un des nœuds et au sein duquel il vit et accède à son individualité ".

37 Ibid., p. 775.

38 Ibid., p. 801. 
relationnel », constituant le « champ relationnel ». D'une certaine manière, sans utiliser les termes "hologrammie ", " hiérarchie enchevêtrée " ou "boucles étranges ", un auteur a incarné ces notions dans un modèle. Ce modèle, qu'il nomme "trialectique » permet de constituer des hiérarchies enchevêtrées en modifiant successivement le niveau auquel interviennent les trois catégories qu'il met en relation.

\section{Le modèle trialectique de Simon Laflamme}

Je tiens d'abord à rappeler que le modèle trialectique a été proposé par Simon Laflamme dès $1992^{39}$. S'il est relativement passé inaperçu ou n'a pas été pris en considération, au moins en France, c'est peut-être à cause de son haut niveau d'abstraction, c'est sans doute aussi parce qu'il remettait en cause des façons dominantes de modéliser. Son ambition est en effet "d'éviter les pièges du positivisme " :

Par ce procédé, aucun des trois éléments essentiels à la structure sociale [il s'agit de la circulation des biens, de celle des idées et de celle des personnes - NDA] ne sera jamais étudié isolément. En aucune façon, donc, la théorie ne pourra reposer sur une simple section du tout, ne pourra s'édifier sans que soit pris en considération un élément en l'absence duquel, finalement, aucun des autres ne pourrait jouer de rôle. [...] Ce procédé permet d'éviter les pièges du positivisme, les rapports ne peuvent plus être livrés par de simples corrélations. De plus, il fournit à l'analyse un instrument taillé à la mesure de l'objet qu'il crée : il ne fragmente pas la dialectique ${ }^{40}$.

Ce modèle est fondé sur l'échange et s'enracine dans « une perspective relationnelle [qui] doit prendre ses distances par rapport à une analyse du social qui se donne pour point de départ la nature des acteurs sociaux ${ }^{41} "$.

C'est dans cet esprit que j'ai choisi de reprendre, pour l'adapter à mon propos, le modèle trialectique de Laflamme.

39 Simon Laflamme, La Société intégrée. De la circulation des biens, des idées et des personnes, op. cit.

40 Ibid., p. 238-239.

41 Ibid., p. 49. 
Dans ce modèle, les relations étudiées sont celles qui existent entre les trois éléments du modèle, mais surtout entre chaque élément et la relation existant entre les deux autres. Ce sont des mesures de « concentration relative » qui permettent de déterminer comment la circulation des biens, des idées ou des personnes structure l'espace social. Par exemple: "les idées s'échangent des personnes. C'est le cas, par exemple, quand les acteurs alternent entre diverses opinions ou quand ils s'identifient à divers discours sociaux. Moins est restreint l'échange [donc, moins il y a concentration de ce point de vue - NDA], moins l'idéologie fractionne la sociétét ${ }^{42}$ ".

Le modèle donne accès à une vision du système sociétal qui n'a nullement besoin d'hypothèses sur la rationalité des acteurs, sur leurs modes de comportement liés à leur position culturelle... Il permet donc de caractériser le « social » sans mettre des acteurs en relation.

La théorie de la société intégrée que propose Laflamme "ne prétend ni être exclusive, ni exhaustive [...] Le constructivisme auquel elle adhère l'empêche d'instituer une connaissance comme vérité absolue » et, malgré ses divers intérêts, " elle n’en demeure pas moins partielle $»^{43}$.

\section{Le modèle trialectique hologrammatique}

Dans le "modèle trialectique hologrammatique ", on reprend l'idée de relation entre trois éléments ou, sans doute mieux, de circulation du champ relationnel entre trois catégories analytiques. On garde aussi l'idée qu'il n'y a pas de prééminence d'une catégorie sur l'autre. Mais on insiste sur l'idée que ces catégories ne sont pas forcément au même niveau. Chacune de ces trois catégories intervient à son propre niveau mais aussi au niveau où se trouvent les autres. Cette idée était déjà présente implicitement, on l'a vu, dans le modèle trialectique de Laflamme : les niveaux interviennent chaque fois que le modélisateur décide d'étudier une catégorie en fonction de la relation entre les deux

\footnotetext{
$42 \quad$ Ibid., p. 243.

43 Ibid., p. 256.
} 
autres, donnant à la première, d'une certaine façon, prééminence provisoire sur les deux autres.

Le caratère hologrammatique du modèle permet d'expliquer comment les catégories sont en même temps homogènes et hétérogènes, à des niveaux différents et pourtant aussi au même niveau, du fait des sauts de niveau qui s'opèrent. La cause essentielle du paradoxe, si difficile à traiter, est liée, selon Barel que je reprends, au fait que les éléments contradictoires qui forment ce dernier sont tous traités comme étant de même niveau. L'introduction de différences de niveau à divers moments de l'analyse permet de prendre en compte explicitement le paradoxe, sans que la cohérence du raisonnement ni celle de la construction du modèle soient mises en cause. Enfin, les changements de sens (causal $v$ s résultant) qui apparaissent permettent de saisir l'intégralité du spectre théorique : dans le modèle général, les pôles peuvent être simultanément causaux et résultants; on l'a vu, dans les principaux courants de pensée en sociologie, ces pôles sont généralement fixés comme étant soit causaux, soit résultants. S'il n'est pas impertinent de fixer ainsi le niveau d'une catégorie analytique dans une théorie ou dans un modèle, le risque est que l'auteur de ceux-ci nous laisse croire que cette modélisation est la seule possible et épuise la totalité de la réalité observable. Ce n'est pas l'incomplétude de la théorie qui est condamnable, c'est la prétention à la complétude... La recherche des conditions minimales d'une théorie générale ne l'est pas en soi non plus, tant qu'elle se mène avec la conscience de ses propres limites.

Ce qui distingue essentiellement ce modèle de celui de Simon Laflamme est le fait que les pôles ne sont plus identiques à ceux du modèle initial. Au lieu des biens, idées et personnes qui sont des éléments échangeables "matériellement " pourrait-on presque dire $^{44}$, il s'agit à la fois d'approches du social, de positions analy-

$44 \quad$ En tout cas, il s'agit de relations qui se représentent sous une forme " presque matérielle » : "Intuitivement, la concentration des biens (et des services) est relative au nombre de personnes qui en font usage, qui en disposent (ou qui y ont recours). Plus est grand le nombre des personnes qui disposent d'une richesse donnée, moins elle est concentrée ", écrit Laflamme dans La Société 
tiques et de catégories analytiques : le pôle «individu " répond à l'approche de la sociologie individualiste; mais, en même temps, il s'agit de l'existence même des individus (terme désignant des personnes ou des groupes, ce que l'on nomme " acteurs " dans les sociologies de l'action) en tant que vecteurs de la vie sociale ("vecteur " me paraît être un terme relativement neutre pour dire que l'individu est à la fois producteur (acteur) et produit (production) de la vie sociale). Le pôle "système " est en écho à l'approche holiste où les structures, les configurations ou encore les modes d'évolution de l'ensemble de la société influencent les actions individuelles; mais le système (également producteur et produit de la vie sociale) n'a de sens que réincorporé à la dynamique sociétale d'ensemble (les pôles et les relations non séparés). Enfin, le pôle "événement » caractérise l'historicité de la société, de l'individu comme du système; mais cette historicité ne représente pas un temps extérieur qui passerait indépendamment des individus et/ou du système. Le temps est tout autant producteur des individus et du système qu'il en est le produit : il est défini par les différences dont parlait Bateson, ce sont les changements dans les individus et dans le système qui sont les marqueurs (ou les indicateurs) du temps qui passe.

Ces trois pôles sont donc à la fois hétérogènes (ils ne sont pas de même niveau, c'est-à-dire qu'ils se situent en des lieux différents de l'explication du social) et homogènes (aucun n'a prééminence sur les autres, aucun ne peut véritablement exister sans les autres); enfin, ils se rabattent les uns sur les autres, du fait du caractère hologrammatique accordé au modèle, pour faire émerger le sens qui gît au sein de la construction.

La production sémantique des termes "individus ", " système " et " événements ", de même que celle des relations entre eux et entre eux et la relation entre les autres termes comprend le fait, à mon avis peu ou pas exprimable analytiquement, que l'individu, le système, l'événement sont tous les uns dans les autres : l'individu est constitué par l'événement (ou la succession des événements) et sa relation avec le système, comme l'événement 
est constitué par l'individu et par la relation existant entre l'individu et le système. Il en va de même pour le système et ses relations avec les deux autres termes.

Le paradoxe de la dialogie de chaque terme et des relations est saisissable dans la mesure où l'on admet que chaque terme est à la fois lui-même et, au moins en partie, les autres. Ce que, analytiquement, nous séparons, faisant ainsi apparaître des « relations entre " est en fait " ontologiquement " unifié ${ }^{45}$.

Le " champ relationnel » constitué de l'ensemble des relations (les flèches du modèle et les pôles) ${ }^{46}$ signifie que ce sont ces relations qui sont productrices des éléments du système. Sans ces relations, les trois catégories I, $S$ et $E$ ne vivraient pas : aucune d'entre elles n'aurait de sens. Que serait un individu non inséré dans une construction sociale ou dans le temps? Et un système ne produisant pas de "parties " et/ou n'étant constitué d'aucune partie? En quoi un "Événement " serait-il événement s'il n'était enchâssé dans les interactions entre individus, individus et système et ne dépendait pas de la qualification que donnent de lui les individus et le système...?

Je viens d'essayer de décrire les fondements et les caractéristiques du modèle que j'appelle « trialectique hologrammatique ». Je vais maintenant en donner une interprétation graphique commentée.

La figure 1 ci-dessous décrit le modèle qui vient d'être succinctement exposé.

45 Si l'on suivait Pribram, on devrait dire que la « réalité ontologique » n'est en fait qu'une construction holonomique que réalise notre cerveau en captant l'énergie des choses : de ce fait, le cerveau, tout à la fois séparerait et rassemblerait, dans une construction holonomique représentant le monde.

46 On pourrait parler, peut-être de façon plus juste, de « circulation du champ relationnel entre les catégories du modèle ». 
Figure 1: Le modèle trialectique et le principe hologrammatique

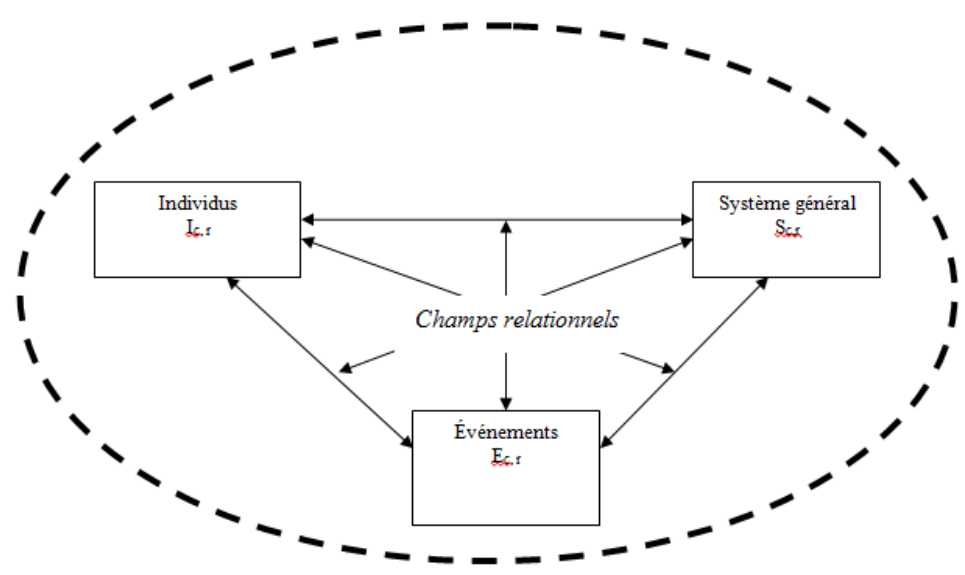

Les niveaux affectant $I, S$ et $E$ se lisent ainsi : $c=$ causal et $r=$ résultant

Les flèches au centre de la structure représentent les relations entre un pôle et la relation entre les deux autres. Ce sont elles qui fondent le modèle trialectique.

Pour l'opérationnalisation du modèle, la forme de celui-ci peut être décrite comme dans la figure 2 ci-dessous.

Figure 2 : Schéma pré opérationnel du modèle

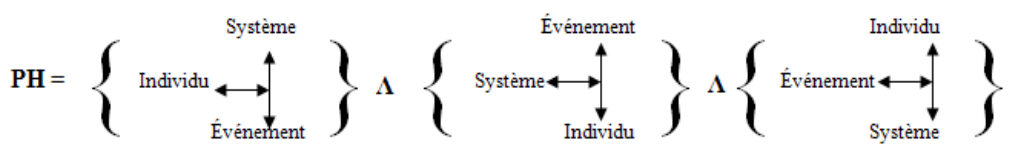

Où :

PH est le phénomène à expliquer

$\Lambda$ est un connecteur logique indiquant que le modèle réside dans la conjonction des trois modules (1), (2) et (3) 
Le signe « = » est métaphorique et signifie seulement que l'explication recherchée réside dans les informations tirées de la conjonction des modules.

\section{Commentaire}

Le modèle est composé de trois modules qui sont reliés entre eux (par le connecteur logique $\Lambda$ ). Chacun de ces modules place une catégorie en relation avec la relation entre les deux autres. À titre d'exemple, le premier module met en scène, pour un phénomène donné (par exemple, le suicide " au travail " ${ }^{47}$, en France et au Canada) et pour un cadre donné (le statut juridique de l'entreprise, par exemple) la question suivante : lorsqu'une personne se suicide " au travail ", dans quelle mesure les caractères particuliers de cette personne sont-elles entrées en résonance avec un environnement spécifique représenté ici par la résultante de l'interaction entre le système et l'événement dans lesquels s'est trouvé inséré l'individu? Cette question suggère que les particularités de la personne peuvent la conduire à l'acte suicide parce que elle interprète (se représente) d'une manière particulière un ou des événements se produisant dans un ou des systèmes qu'elle considère comme ses coordonnées de vie. Qu'ainsi, ce ne sont pas ses seules caractéristiques personnelles (être dépressive, suicidaire...), pas plus que seulement celles du système (être inégalitaire, injuste...) ou événementielles (difficultés matérielles, affectives...) qui interviennent comme causes, mais le faisceau des éléments représentés dans le modèle et, dans ce faisceau, la combinaison particulière des catégories entre elles.

La conjonction des trois modules du modèle permet, par exemple par l'intermédiaire d'indices de concentration des catégories les unes par rapport aux autres, de brosser un tableau de l'univers dans lequel se mouvait la personne qui s'est suicidée. Le résultat attendu est que si chacune des catégories est potentiellement explicative ou causale, aucune n'est sans doute obligatoirement dominante comme cause, encore moins exclusive.

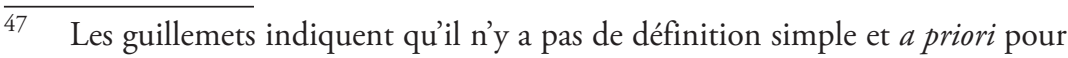
le terme " au travail » en ce qui concerne le suicide. C'est évidemment une question qui sera traitée lors de l'application du modèle. 
La mise en oeuvre concrète du modèle dépasse le cadre de cette réflexion. Elle consistera, sur le thème choisi, à partir d'enquêtes, de rapports et d'articles de périodiques concernant le phénomène étudié, depuis une dizaine d'années, de tenter de mettre en évidence les relations existant entre les trois catégories, en fonction de variables potentiellement discriminantes : région, culture locale (laïque ou religieuse...), sexe, âge des personnes suicidées, type d'entreprise (publique, privée)...

L'objectif sera de faire apparaître si le phénomène du suicide au travail est explicable par l'une ou l'autre de ces caractéristiques ou par leur combinaison telle qu'organisée par le modèle et, de façon plus générale, si ce phénomène est principalement justiciable d'une analyse holistique, individualiste, synchronique ou diachronique, l'hypothèse de départ étant que ces trois dimensions de la vie d'une société doivent être intimement mêlées pour comprendre ce qui se passe vraiment.

\section{Conclusion}

Quelle conclusion peut-on tirer de cette réflexion? Celle-ci me semble montrer que les querelles entre les principaux courants théoriques en sociologie peuvent être dépassées par une analyse relationnelle et une perspective hologrammatique.

Chacun de ces grands courants de pensée porte en lui des éléments de compréhension des phénomènes sociétaux. Chacun éclaire un pan des processus qui forment ce que nous appelons "la société ". Mais, en le faisant à la manière préconisée par Descartes, en séparant ces éléments, il perd une dimension essentielle que j’ai appelée ici « hologrammatique » en reprenant le terme à Edgar Morin. Comme le dit Castoriadis « une fois la séparation commise, il sera à jamais impossible de ressaisir ce dont il s'agit ${ }^{48}{ }^{\prime}$.

48 Cornélius Castoriadis, op. cit., p. 165. Durkheim lui-même écrivait, par exemple, dans Les Règles de la méthode sociologique : "les phénomènes sociaux sont beaucoup trop complexes pour que, dans un cas donné, on puisse retrancher l'effet de toutes les causes moins une ", Paris, Presses universitaires de France, 1999 [1894], p. 128. 
Dans une sociologie relationnelle, puisque ce ne sont plus les objets, les choses, les êtres qui importent pour l'analyse, mais le champ relationnel duquel ils émergent, dans lequel leur " êtresoi » comme leur " être aux autres " prend consistance et sens, la question ne se pose plus dans les mêmes termes. Dans le cas du modèle que j'ai proposé, les catégories analytiques, certes réduisent le champ de la réflexion, mais sont suffisamment larges : ce faisant, elles ne sont pas vagues, mais denses, riches d'une multitude d'occurrences que l'on peut retenir dans des contextes empiriques divers. Elles peuvent embrasser, non pas l'intégralité du social, mais ce qui en fait la profondeur, y compris la profondeur historique. La revue que j'ai proposée des principaux courants théoriques depuis les débuts de la sociologie montre que ces trois catégories ont, à des titres divers, été centrales dans leurs analyses. Que certaines sont absentes dans certains courants, ou minorées, ou encore ramenées aux précédentes ou à l'une d'entre elles (l'événement, chez Boudon, par exemple, apparaissant comme un effet de composition, c'est-à-dire la résultante des actions combinées des individus). Elle montre également que, très souvent, un courant théorique donne à l'une de ces catégories la prééminence sur les autres : chez les fondateurs, l'individu et le système peuvent être causaux, mais l'événement ne l'est jamais, montrant que l'analyse est surtout synchronique, même si des interrogations et des affirmations peuvent se situer dans la diachronie, tel le « désenchantement du monde " chez Weber. Dans les fonctionnalismes, c'est le système qui est causal, de même que dans les structuralismes. Dans les théories de l'action, c'est l'individu, sauf chez Crozier pour qui l'individu n'est pas vraiment séparable du système. Il faut aller vers les théories systémiques pour que les trois catégories apparaissent potentiellement opérantes. Bien entendu, tous les auteurs contemporains se sont ralliés à l'idée que l'individu et le système sont co-producteurs du social. Mais chez les interactionnistes ou encore chez Boudon, l'individu est plus déterminant que le système. 
La sociologie fondée sur la théorie des systèmes complexes telle que définie par Morin professe que l'on doit rassembler, conjoindre les catégories et non les disjoindre. Mais peu de travaux empiriques sont venus illustrer ou appuyer cette importante profession de foi ${ }^{49}$. Le Réseau national des systèmes complexes, pour sa part, s'est orienté davantage vers la simulation multi agent et a tendance, de ce fait, à redonner à l'individu une place centrale, puisque c'est à partir d'une psychologie de convention (Weber) que l'on modélise et que les interactions des agents ou acteurs modifient le système qui n'est, dans le modèle SMA, qu'un cadre dans lequel on fait agir ces agents ${ }^{50}$.

Mon but n'était pas, dans cet article, de classer hiérarchiquement les théories et de distribuer des bons et des mauvais points. Toutes ces approches présentent un intérêt certain. Dans la mesure où chacun a raison au moins sur une partie de l'analyse, dans celle aussi où des éléments d'idéologie s'incorporent à celle-ci, puisqu'il n'est pas équivalent de dire que c'est l'individu ou le système qui agit et est donc responsable (glissement, certes, mais compréhensible et observable), les débats ne peuvent qu'être à la fois durs et interminables, sans doute aussi stériles.

Une sociologie relationnelle couplée à la théorie de la complexité, me paraît être une piste féconde pour parvenir à sortir de cette situation. L'application qui en sera faite à partir du modèle proposé devrait dire si elle est féconde ou non.

49 Parmi quelques rares auteurs qui s'y sont essayés, on peut citer Pascal Roggero, notamment dans De la complexité des politiques locales, Paris, L'Harmattan, 2005.

50 Bien que ce cadre soit de plus en plus raffiné et, dans les développements actuels, soit doté de possibilités de transformations liées aux interactions des agents entre eux. 


\section{Bibliographie}

Guy Bajoit, "Le Concept de relation sociale ", Nouvelles Perspectives en sciences sociales, vol. 5, $\mathrm{n}^{\circ}$ 1, 2009, p. 51-65.

Bajoit, Guy, Pour une sociologie relationnelle, Paris, Presses universitaires de France, 1992.

Barel, Yves, "L'Idée de système dans les sciences sociales ", Esprit, $\mathrm{n}^{\circ} 1$, janvier 1977, p. 69-82.

Bohm, David, La Danse de l'esprit ou le sens déployé, La Varenne-Saint Hilaire, Seveyrat, non daté.

Bouchard, Pierre, "Théorie de l'action et parcours de vie ", Nouvelles Perspectives en sciences sociales, vol. $1, \mathrm{n}^{\circ} 2,2006, \mathrm{p} .67-114$.

Castoriadis, Cornelius, Les Carrefours du labyrinthe, tome 1, Paris, Seuil, 1978.

Chazel, François et Jacques Coenen-Huther, "La Théorie sociologique générale en question ", Revue européenne de sciences sociales, tome XLVI, 2008, n 140 , p. 5-14.

Concert'Eau sur le Site <concerteau.ecobag.org/>

Daré, William's, Christine Fourrage et Ibrahima Diop Gaye, « Positionnement des sociologues dans la démarche de modélisation Domino ", Nouvelles Perspectives en sciences sociales, vol. 2, n 2, 2007, p. 103-126.

Donati, Pierpaolo, "La Relation comme objet spécifique de la sociologie ", Revue du MAUSS, vol. 2, n 24, 2004, p. XX-XX, en ligne sur <www. cairn.info/load_pdf.php?ID_ARTICLE=RDM_024_0233>, consulté le 19072012.

Elias, Norbert, La Société des individus, Paris, Fayard, 1991 [1987].

Emirbayer, Mustafa, "Manifesto for a Relational Sociology ", American Journal of Sociology, vol. 103, n² 2, 1997, p. 281-317.

Gervais, Roger, Presse et Mondialisation : étude comparée franco-canadienne, Thèse de doctorat, Université de Toulouse 1 Capitole, 2009.

Girard, Mélanie, «Éléments de critique des théories de l'action ", Nouvelles Perspectives en sciences sociales, vol. $3, \mathrm{n}^{\circ} 1,2007, \mathrm{p}$. 47-60.

Girard, Mélanie, Contribution à la critique des théories de l'action. Intention et émoraison, Thèse de doctorat en sociologie, Toulouse, Université des Sciences Sociales, Toulouse 1 Capitole, 2009.

Hofstadter, Douglas, Gödel, Escher, Bach. Les brins d'une guirlande éternelle, New York, Basic Books Inc. Publishers, 1979 (traduction de Jacqueline Henry et Robert French, InterÉditions, 1985). 
Laflamme, Simon, Communication et émotion. Essai de microsociologie relationnelle, Paris, L'Harmattan, coll. «Logiques sociales », Paris 1995.

Laflamme, Simon, La Société intégrée. De la circulation des biens, des idées et des personnes, New York, Peter Lang, 1992.

Morin, Edgar, La Méthode 3. La connaissance de la connaissance, Paris, Seuil, 1986.

Roggero, Pascal et Christophe Sibertin-Blanc, "Quand des sociologues rencontrent des informaticiens : essai de formalisation, méta modèle, modélisation et simulation des systèmes d'action concrets ", Nouvelles Perspectives en sciences sociales, vol. $3, \mathrm{n}^{\circ} 2,2008, \mathrm{p} .41-81$.

Roggero, Pascal, De la complexité des politiques locales, Paris, L'Harmattan, 2005.

Schumpeter, Joseph, Histoire de l'analyse économique, Paris, Gallimard, 1954.

Vautier, Claude, "La Longue marche de la sociologie relationnelle ", Nouvelles Perspectives en sciences sociales, vol. 4, $\mathrm{n}^{\circ}$ 1, 2008, p. 77-106. 